\title{
Histochemical changes in neocortex and corpus callosum after intracranial injection
}

\author{
N. ROBINSON ${ }^{1}$ \\ From the Department of Chemical Pathology, Institute of Neurology, The National Hospital, \\ Queen Square, London
}

The penetration of a syringe needle or electrode into the brain, causing localized physical changes such as a high compression front with displacement of whole cells and other cells suffering from various degrees of temporary distortion, would be expected to produce disturbances in metabolism. Subsequent intracranial injection of small quantities of fluid may produce changes in $p \mathrm{H}$, electrolyte concentration, ionic strength, temperature, and the disequilibrium of colloidal suspensions. Less clearly defined effects on metabolism due to inflammatory processes and trauma would also be likely to occur.

This paper reports the effects of intracranial penetration of a microneedle and subsequent injection of $5 \mu \mathrm{l}$. physiological saline on the metabolic activities of enzymes controlling important metabolic pathways in the brain. Two areas were investigated-namely, the cerebral cortex and the corpus callosum. Following injection, the animals were allowed to recover and observations on enzymes of glucose, thiamine and phosphate metabolism, amine oxidation, and acetylcholinesterase within nerve cells and glia during the period of 31 days post-operation were made by histochemical techniques.

\section{MATERIALS AND METHODS}

Adult male Lister rats weighing between 250 and $300 \mathrm{~g}$ were anaesthetized with ether, the hair shaved off the scalp, and the animal secured in a stereotaxic apparatus. Under aseptic conditions a sagittal incision was made, the skull exposed and scraped clean. The dura mater was exposed by drilling a burr-hole at Area 4 of the motor cortex (Krieg, 1946) and a sterilized specially drawn fine glass microneedle (less than $200 \mu$ in diameter) was introduced to the predetermined depth. Using a Hamilton $100 \mu 1$. syringe, $5 \mu 1$. isotonic saline solution were injected over a period of $10 \mathrm{~min}$ by a motor-driven micrometer. The syringe needle was withdrawn, 300,000 u. Penidural given intraperitoneally, and the skin incision

1Present address: Department of Anatomy, The London Hospital Medical College, Turner Street, London E.1. closed with silk suture material before the animals recovered. In the second series of experiments the microneedle was lowered into the corpus callosum at the same surface co-ordinates as in the first series before injection of $5 \mu 1$. fluid.

The animals were killed at $1,2,3,5,7,14,21$, and 31 days after operation. The brains were removed, rapidly frozen, and fresh-frozen sections 10 to $12 \mu$ thick cut on a Slee cryostat. The techniques for demonstrating enzyme activities have been described (Robinson, 1969a); the enzymes were $\mathrm{NADH}_{2}$-diaphorase, succinate, glucose 6 -phosphate and $\alpha$-glycerophosphate dehydrogenases, 5'-nucleotidase, adenosine triphosphatase, thiamine pyrophosphatase, acid and alkaline phosphatase, acetylcholinesterase, and monoamine oxidase.

\section{RESULTS}

Intracranial injection had no apparent effect on the behaviour of the rats up to 31 days. Animals had recovered and were walking within $30 \mathrm{~min}$ after operation.

The patterns of localization and intensity of the enzymes examined in the normal rat brain have been described (Robinson and Eayrs, 1968). Only the enzyme changes seen in the neocortex and corpus callosum at the site of intracranial injection at time intervals up to 31 days post-injection are described. The furrow ploughed by the microneedle was seen as a region of complete tissue necrosis; adjacent to this was a narrow band of traumatized tissue on either side less than $200 \mu$ wide.

TWENTY-FOUR HOURS POST-INJECTION Oxidative enzymes, 5'-nucleotidase (Fig. 1) and acid phosphatase showed changes in activity within this time. $\mathrm{NADH}_{2}$-diaphorase was raised in the cytoplasm of some glial and nerve cells in traumatized cortex. Acid phosphatase was above normal in nerve cell bodies and glial cells.

In the corpus callosum raised glial cell $\mathrm{NADH}_{2}-$ diaphorase activity was accompanied by swelling and loss of cytoarchitecture; some nerve fibres 


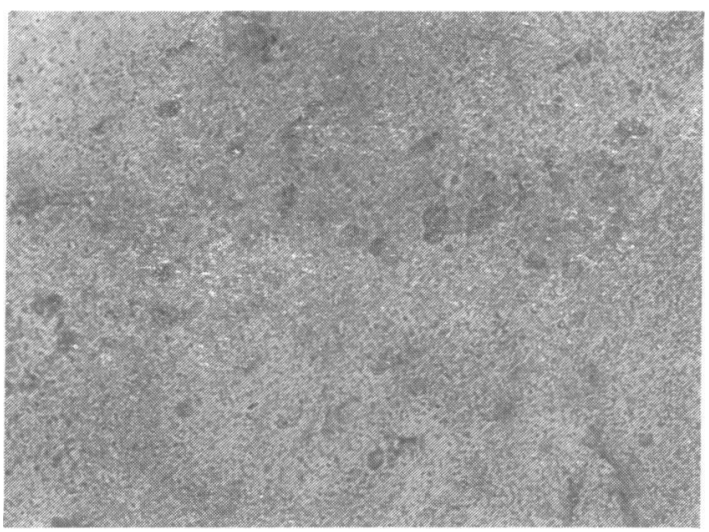

FIG. 1. Twenty-four hours. Slightly raised 5'-nucleotidase activity in nerve cell perikarya adjacent to site of injection. $\times 24$.

adjacent to necrosis showed a discrete weak reaction not normally seen. Fewer glial cells showed raised succinate and $\alpha$-glycerophosphate dehydrogenase activities (Fig. 2) than seen in the cortex. A striking increase in acid phosphatase activity was seen in nerve fibres, normally exhibiting no acid phosphatase activity (Fig. 3); the fibres showed a moderate intermittent reaction with swollen hyperactive glial cells distributed among them. Glial cell 5'-nucleotidase activity was raised in a small well-defined area, but immediately beyond this region the enzyme appeared normal whereas acid phosphatase was raised.

TWO DAYS POST-INJECTION $\mathrm{NADH}_{2}$-diaphorase had further increased, but glucose 6-phosphate dehydrogenase activity had decreased within cortical nerve cells (Fig. 4); in these cells abnormal acid phosphatase and 5'-nucleotidase activity showed stronger reactions.

An increase in glial cell population exhibiting raised $\alpha$-glycerophosphate, succinate and glucose 6-phosphate dehydrogenase was apparent in the corpus callosum.

THREE DAYS POST-INJECTION In the cortex an increase in the population of swollen astrocytes with loss of processes and also of round cells was accompanied by more intense $\mathrm{NADH}_{2}$-diaphorase, $\alpha$-glycerophosphate, glucose 6-phosphate and succinate dehydrogenase activities than seen at two days; a discrete enzyme activity was also apparent along some axons. A pallor in the neuropil, where activity of this enzyme was normally moderate, resembled the reaction seen in spongy degeneration (Robinson, 1969b). ATPase activity, previously normal, was raised in capillaries and in the cytoplasm of some nerve cells adjacent to necrosis.

The corpus callosum exhibited a similar pattern of oxidative enzyme activity to that seen at one day. Acid phosphatase appeared as discrete elongated particles with loss of cytoarchitecture but resembling deformed oligodendrocytes, probably macrophages (Strong and Elwyn, 1953) lying between fibres, the activity rapidly diminishing away from tissue necrosis. ATPase activity, previously showing negligible change, was more prominent than normal in this region at three days. The distribution of abnormal 5 '-nucleotidase activity showed no further change from $24 \mathrm{hr}$.

FIVE DAYS POST-INJECTION Some enzyme abnormalities were seen at this time which had previously appeared normal. In traumatized tissue formazan particles from the monoamine oxidase reaction were localized within round bodies of various sizes scattered at random; adjacent to this area were fragmented axons with strong activity. Further away

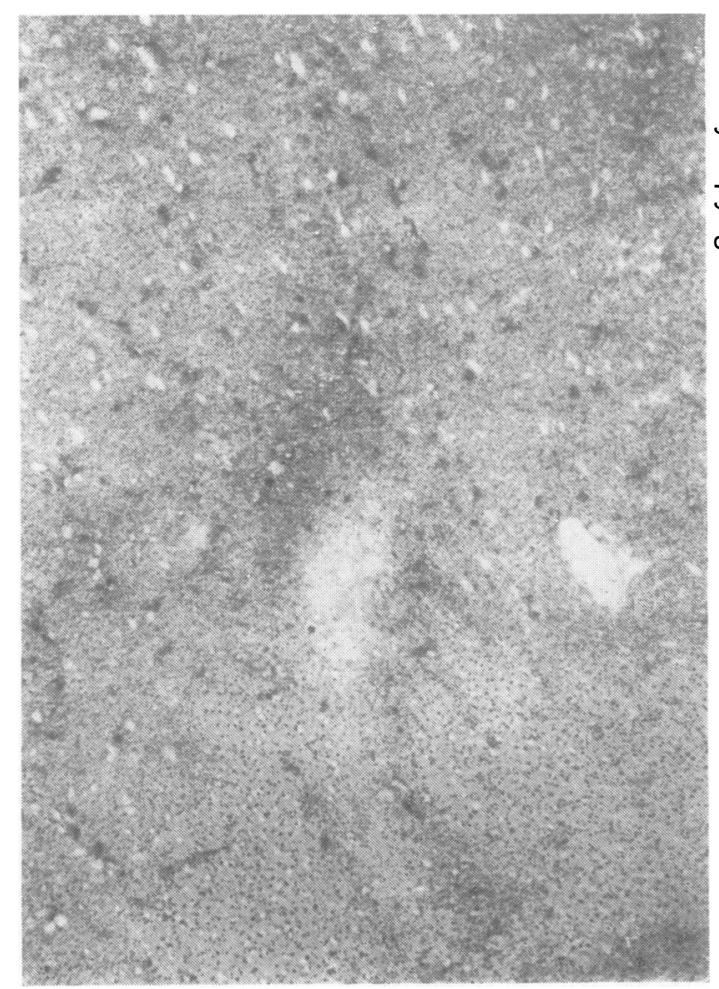

FIG. 2. Twenty-four hours. Raised $\alpha$-glycerophosphate dehydrogenase activity in cortical glial cells. Lower right hand side shows relatively normal activity in subjacent white matter. $\times 100$. 


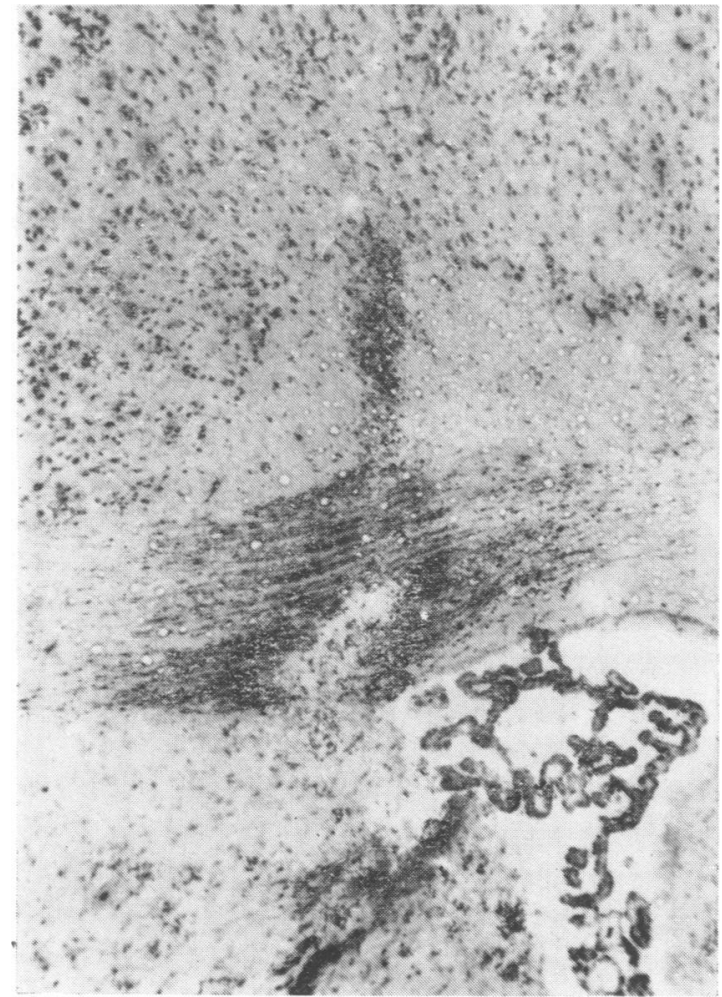

FIG. 3. Twenty-four hours. High acid phosphatase activity in glial cells and nerve fibres of the corpus callosum. $\times 40$.

from the lesion reactive glial cells contained numerous small formazan particles and axons were more intensely stained than normal. The only change seen in acetylcholinesterase was a loss of reaction within nerve cells in the traumatized region and a slightly raised nerve cell activity adjacent to this area (Fig. 5). In neuropil a raised thiamine pyrophosphatase appeared similar to the diffuse slightly raised intensity of ATPase. In traumatized tissue thiamine pyrophosphatase was normal in the vascular endothelium but with some loss in the adventitia. Fragmentation and loss of this enzyme was seen in Golgi lamellae and cytoplasmic membranes of nerve cells adjacent to traumatized tissue.

ONE WEEK POST-INJECTION The high activity of $\mathrm{NADH}_{2}$-diaphorase seen in some nerve cells at $24 \mathrm{hr}$ persisted but the loss in activity within neuropil appeared partly restored. Both $\mathrm{NADH}_{2}$-diaphorase and $\alpha$-glycerophosphate dehydrogenase showed the same intense activity in swollen astrocytes and macrophages in both neocortex and corpus callosum seen previously. Activity of 5 '-nucleotidase was intense

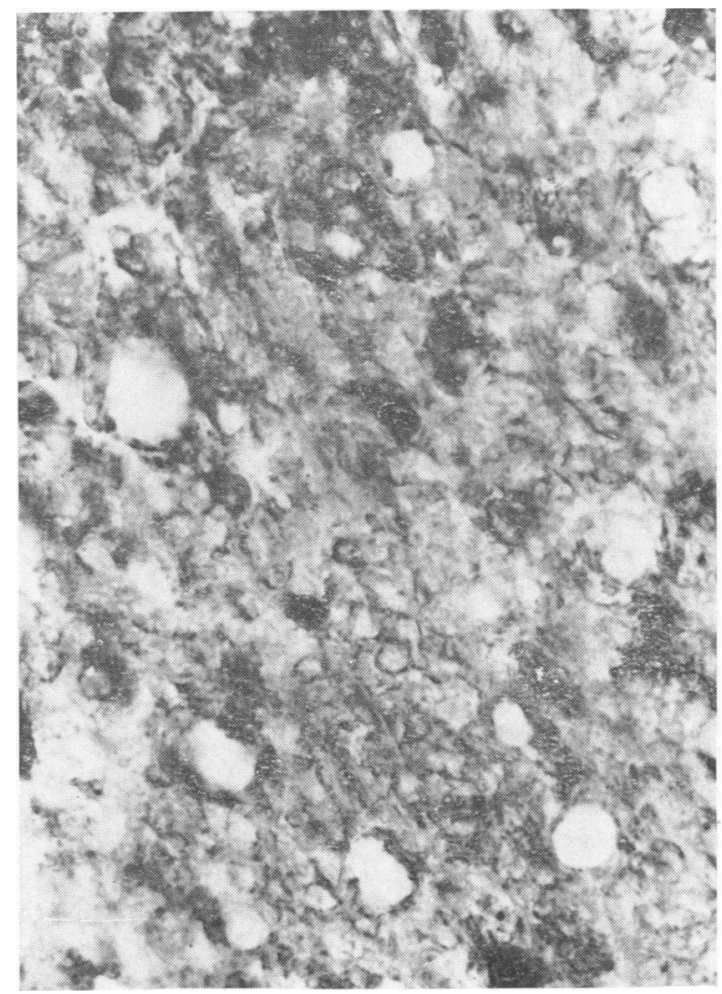

FIG. 4. Two days. Raised glucose 6-phosphate dehydrogenase activity in some glial cells but no activity in nerve cells. $\times 240$.

in swollen hyperactive glia, capillary walls, and in some nerve cell perikarya but absent in others (Fig. 6 and compare with Fig. 1). A raised ATPase reaction was more in evidence in the neocortex at this stage than at three days; isolated nerve cells and numerous glial cells in traumatized tissue were prominently stained, but immediately outside this zone the reaction was normal. Alkaline phosphatase activity was raised in blood vessels adjacent to necrosis (Fig. 7).

In the corpus callosum 5'-nucleotidase activity was raised where fluid appeared to have been injected. In this region cellular localization of $5^{\prime}$-nucleotidase was poor, but in degenerating fibres and compound granular corpuscles it was seen as minute discrete deposits.

THREE WEEKS POST-INJECTION Oxidative enzymes in the neocortex continued to be raised in glial cells and macrophages within and adjacent to traumatized tissue, but $\mathrm{NADH}_{2}$-diaphorase in nerve cells and capillaries was less prominent than before. Many large nerve cells in and near traumatized tissue 


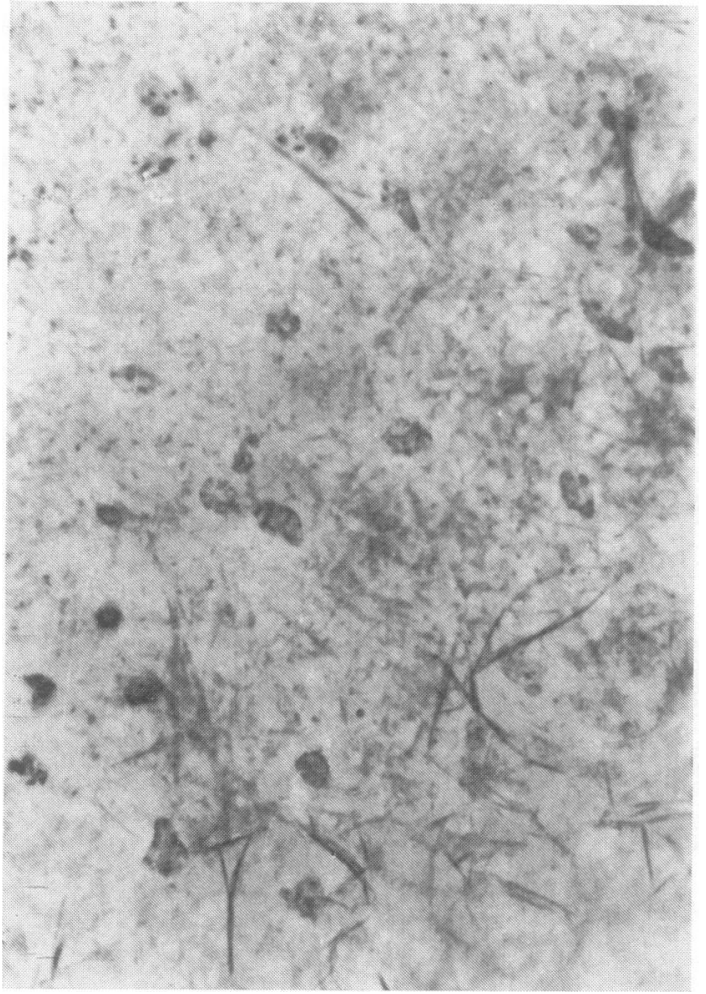

FIG. 5. Five days. Raised acetylcholinesterase activity in nerve cells adjacent to traumatized tissue. $\times 280$.

showed a lower but fragmented acid phosphatase reaction (Fig. 8), smaller nerve cells were more normal but also with some loss in localization; glial cell acid phosphatase activity was less prominent than previously. Alkaline phosphatase exhibited the same raised activity within a larger number of capillaries than were seen after one week. Within this region an unusually prominent ATPase activity was seen in swollen glial cells and macrophages and in damaged axons; nerve cell bodies were difficult to identify (Fig. 9). Swollen deformed nerve cells with intense acid phosphatase activity were prominent amid numerous hyperactive macrophages and interweaving bundles of naked axons of variable diameter (Fig. 10). Acid phosphatase in these axons appeared as discrete granules regularly spaced along the fibre.

Raised activities of several enzymes (normally inactive in myelin) in fragmented fibres of the corpus callosum were still apparent. Numerous glial cells appeared to have an enlarged displaced nucleus, both these and macrophages exhibited a strong $\alpha$ -

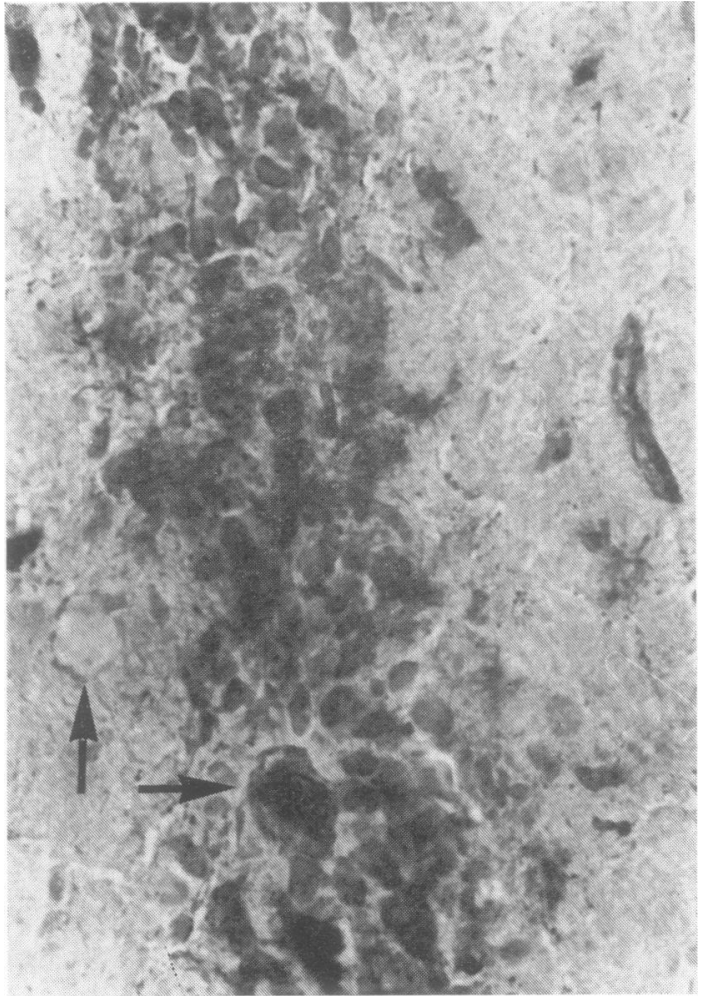

FIG. 6. One week. Intense 5'-nucleotidase activity in glial cells, but variable in nerve cells (arrowed) within a narrow well-defined region of neocortex. $\times 250$.

glycerophosphate dehydrogenase reaction which was poorly localized. The few capillaries seen in the corpus callosum exhibited a higher alkaline phosphatase activity than normal. Acetylcholinesterase, monoamine oxidase, and thiamine pyrophosphatase showed no marked changes from those seen at five days.

THIRTY-ONE DAYS POST-INJECTION At this time traumatized tissue had a distinct appearance of scarring. In the small affected region of the neocortex oxidative enzymes and 5'-nucleotidase activity continued to exhibit some pallor in the neuropil and in axons. Hyperactive swollen astrocytes and macrophages which had previously exhibited strong enzyme reactions appeared fewer in number and exhibited more normal oxidative enzyme, $5^{\prime}$-nucleotidase and ATPase activities (Fig. 11), but some large isolated glial cells still showed intense 5 -nucleotidase and acid phosphatase reactions. 
Enzyme reactions in a large population of cells within the corpus callosum showed no marked changes from those seen 10 days previously.

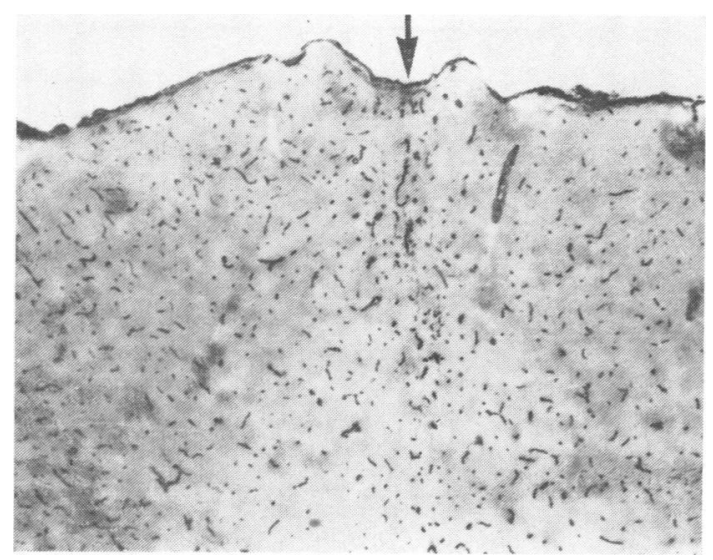

FIG. 7. One week. A greater prominence of blood vessel alkaline phosphatase activity than normal in neocortex. $\times 27$.

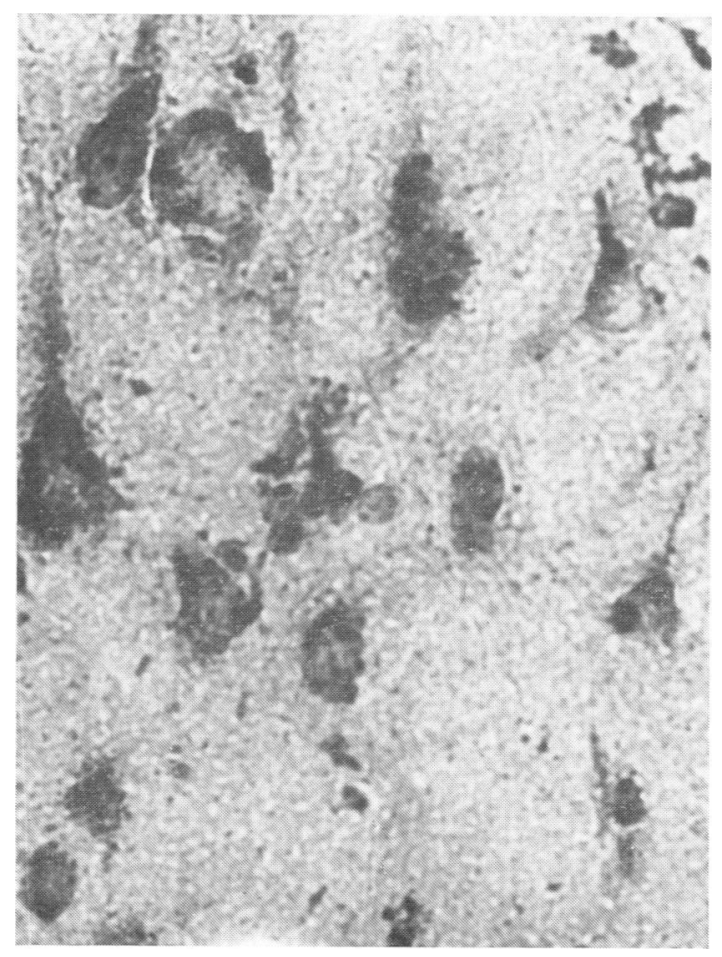

FIG. 8. Three weeks. Fragmented and lower nerve cell acid phosphatase activity. $\times 380$.
DISCUSSION

Penetration of the microneedle into both the neocortex and corpus callosum of the rat brain produced a remarkably discrete and limited area of complete tissue necrosis; adjacent to this on either side a narrow band of traumatized tissue was apparent. Further tissue damage after injection of $5 \mu l$. physiological saline delivered over $10 \mathrm{~min}$ was limited to an area extending in depth very little beyond the width of the needle. This evidence is supported by the changes seen in enzyme activity within nerve cells and neuroglia reflecting alterations in the metabolism within these cells.

In comparing the enzyme response of the various intracellular organelles in the neocortex to disturbances after intracranial injection, increases in the nerve cell cytoplasmic mitochondrial oxidative enzymes (except monoamine oxidase) were substantial within 24 hours when compared with the normal controls. Evidence from the strong oxidative enzyme reactions suggests that increased demands were being made on oxidative metabolism and that the mitochondria had retained morphological

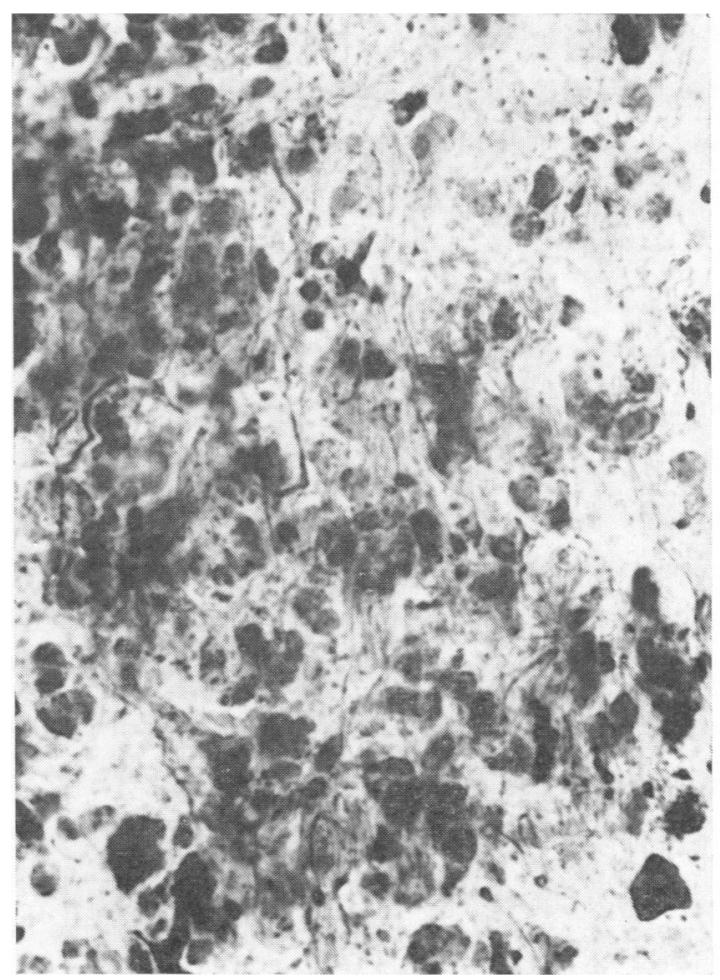

FIG. 9. Three weeks. Unusually high ATPase activity in swollen glia and damaged axons in neocortex. $\times 380$. 


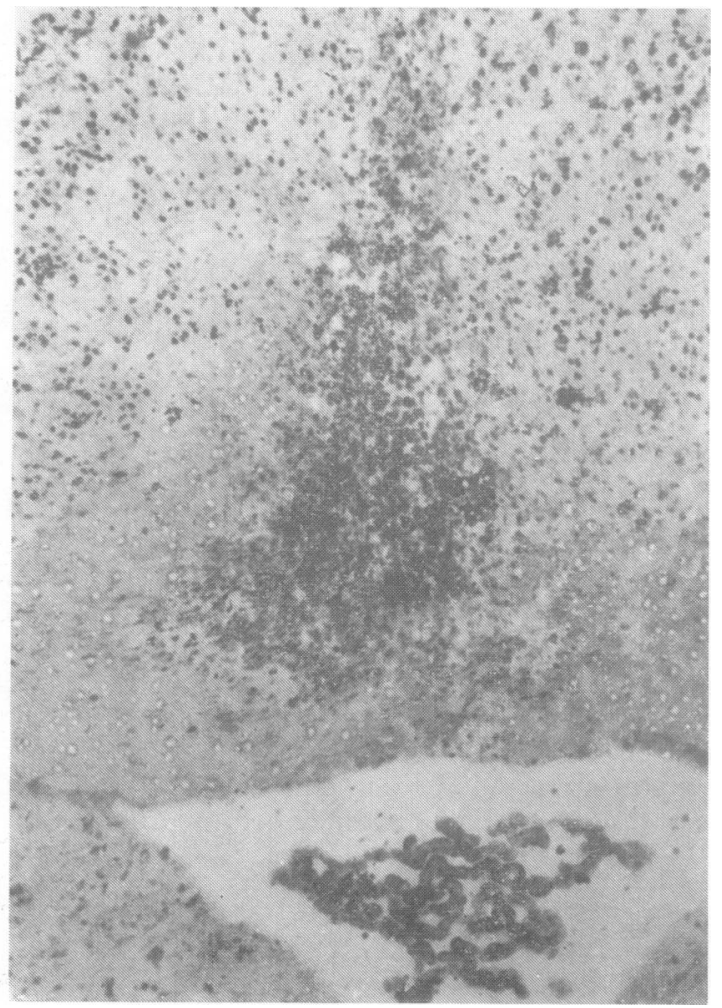

FIG. 10. Three weeks. Numerous hyperactive glia but fewer myelin fragments with intense acid phosphatase activity were seen in the corpus callosum at this time (compare with Fig. 3). $\times 40$.

integrity. In contrast with the marked alterations in activity of the oxidative enzymes in these organelles, the mitochondrial monoamine oxidase and acetylcholinesterase activity, localized at nerve endings, reacted very little to tissue damage. The sensitivity of some oxidative enzymes in the neuropil to injury was seen by the pallor in $\mathrm{NADH}_{2}$-diaphorase and $\alpha$-glycerophosphate dehydrogenase staining within 24 hours, although a reaction persisted in the blood vessels. Similar responses of these enzymes have been observed after application of cold (Rubinstein, Klatzo, and Miquel, 1962) and in CreutzfeldtJakob disease (Friede and DeJong, 1964). Astrocytes in the neocortex adjacent to injury showed an intense activity of oxidative enzymes; these cells were more responsive than others to tissue damage. The increase in activity of oxidative enzymes within the astrocytes indicated that the main pathways of glucose metabolism-namely, glycolysis and the pentose and tricarboxylic acid cycles-were involved,

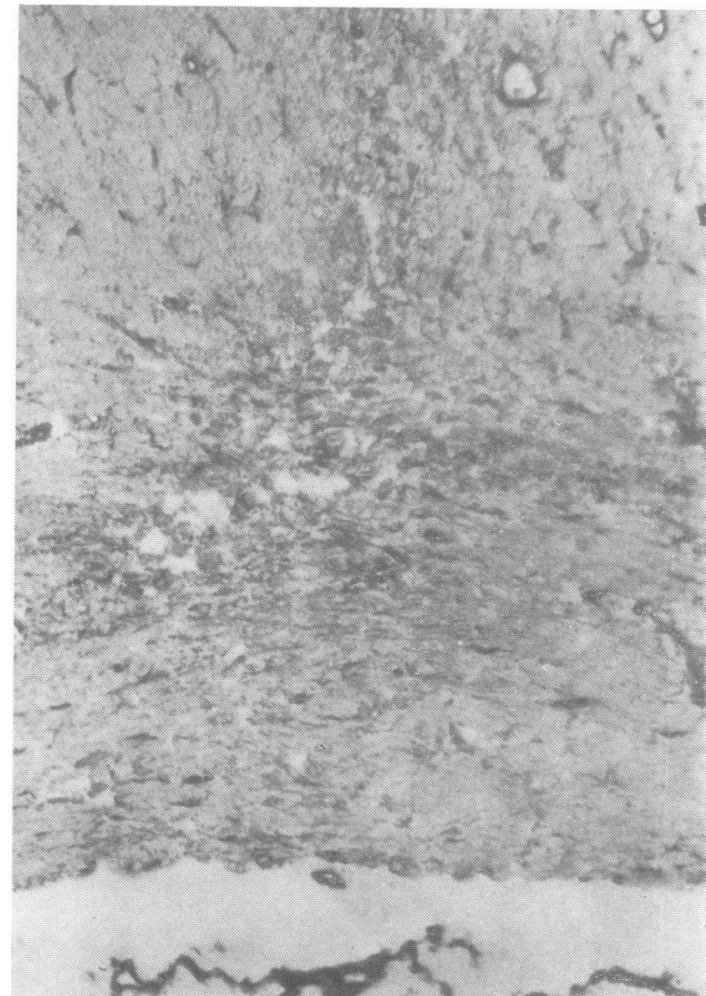

FIG. 11. Thirty-one days. A lower ATPase actvity in glial cells and naked axons in the corpus callosum and neocortex than seen at three weeks (Fig. 9). $\times 100$.

although the response of the enzymes in these metabolic pathways within the reactive cells differed. The evidence in this and earlier studies (Friede, 1962a; Rubinstein et al., 1962) does suggest that the active microglia and hypertrophic astrocytes in both neocortex and subjacent white matter have a substantial metabolic turnover soon after cerebral injury. Nerve fibres did not show the marked changes for most enzymes exhibited by other organelles; however, a raised 5 '-nucleotidase activity was apparent and the varicose distribution of monoamine oxidase seen in normal fibres (Robinson, 1967) was depleted.

Progress in the changes in activity with time of the enzymes catalysing the release of phosphate showed distinct variations. The strong lysosomal acid phosphatase reaction, seen in glial cells bordering tissue necrosis, demonstrated an early rapid and continuous response to injury; the same abnormally high activity has been reported at necrotic regions of 
cerebral arteriosclerosis (Friede, 1962b). The mitochondrial ATPase response did not parallel the mitochondrial oxidative enzymes also concerned with energy metabolism; the marked rise in ATPase activity was about six days later and persisted throughout the following 24 days. The substrate (thiamine pyrophosphate) of the enzyme thiamine pyrophosphatase is rich in brain where it is known to be more resistant to depletion in a thiaminedeficient diet than in other organs (McIlwain, 1959). The enzyme, localized mainly in the Golgi apparatus and cytoplasmic membranes of the cortical nerve cells, appeared comparatively insensitive to a disturbed environment after injury, since only a minor change in its activity was seen at the fifth day postinjection.

Acetylcholinesterase, localized at nerve endings, has received little attention in pathological studies; in this investigation it showed only a minor increase in activity adjacent to traumatized tissue at the fifth day.

Another enzyme, monoamine oxidase, did not exhibit any persistent marked response to injury after intracranial injection, although some disturbance in its distribution along axons, seen as varicosity, was apparent.

Some enzyme alterations in the corpus callosum differed from those seen in the neocortex. The most prominent change in the corpus callosum seen after one day was in acid phosphatase activity within oligodendrocytes and swollen microglia; other enzymes showing increased activity in the neocortex (NADH $_{2}$-diaphorase, succinate, and $\alpha$-glycerophosphate dehydrogenases) exhibited negligible reactions as in normal tissue. Where fibres had been severed by the microneedle an intense acid phosphatase reaction was apparent by five days, receding in intensity away from the zone, and giving the impression of 'damming up' of the enzyme as though migration along the nerve fibres had been interrupted. Whether this enzyme, mainly but not entirely located within lysosomes, undergoes axonal flow in the corpus callosum is not known but experiments to confirm this, or otherwise, are planned.

There are fewer enzyme changes in the corpus callosum compared with those seen in the neocortex from one week onwards but there was an increased population of glial cells in which the abnormal enzyme activities were mainly localized.

The histochemical evidence showed that the response of the enzymes was mainly in tissue that had suffered mechanical damage adjacent to the pathway traversed by the needle. Where $5 \mu$ l. fluid had been injected over a period of $10 \mathrm{~min}$ differences in enzyme activities from the normal were much less.

\section{SUMMARY}

Changes in activities of some enzymes controlling important metabolic pathways of the CNS have been examined over 31 days post-intracranial injection of $5 \mu \mathrm{l}$. physiological saline into the motor cortex and corpus callosum of the rat.

The early responses of these enzymes to trauma varied in the intensity of their activities and the rapidity with which they showed increases, but some enzymes (acetylcholinesterase, monoamine oxidase, and thiamine pyrophosphatase) exhibited no early changes.

Within 24 hours nerve cells showed raised dehydrogenase activities, glial cells exhibited increased acid phosphatase and myelinated fibres high acid phosphatase and $5^{\prime}$-nucleotidase activities. At three days an increased glial cell population exhibited further increases in enzyme activities; ATPase was also raised. At five days slight disturbances in monoamine oxidase, acetylcholinesterase and thiamine pyrophosphatase were observed. At seven days alkaline phosphatase was more prominent in an increased population of capillaries than normal. Between seven and 21 days no further significant enzyme changes were apparent but some loss in localization was evident. At 31 days, oxidative enzymes and some phosphatases in the neocortex were less prominent; the corpus callosum retained an abnormally large glial cell population, with raised acid phosphatase activity in particular.

The author thanks the 'Action for the Crippled Child' Fund for financial assistance.

\section{REFERENCES}

Friede, R. L. (1962a). The cytochemistry of normal and reactive astrocytes. J. Neuropath. exp. Neurol., 21, 471-478.

- (1962b). An enzyme histochemical study of cerebral arteriosclerosis with some data on the pathogenesis of periarterial scars. Acta neuropath. (Berl.), 2, 58-72.

$\longrightarrow$, and DeJong, R. N. (1964). Neuronal enzymatic failure in Creutzfeldt-Jakob disease. A familial study. Arch. Neurol. (Chic.), 10, 181-195.

Krieg, W. J.S. (1946). Accurate placement of minute lesions in the brain of the albino rat. Quart. Bull. Northw. Univ. med. Sch., 20, 199-208.

McIlwain, H. (1959). Biochemistry and the Central Nervous System, 2nd edn., p. 135. Ch archill: London.

Robinson, N. (1967). Histochemistry of monoamine oxidase in the developing rat brain. $J$. Neurochem., 14, 1083-1089.

— , and Eayrs, J. T. (1968). Histochemical study of the cerebral cortex in rats thyroidectomised at birth. Brain Res., 9, 351-362.

- (1969a). Histochemistry of human cervical posterior root ganglion cells and a comparison with anterior horn cells. J. Anat. (Lond.)., 104, 55-64.

(1969b). Creutzfeldt-Jakob's disease: a histochemical study. Brain (in press).

Rubinstein, L. J., Klatzo, I., Miquel, J. (1962). Histochemical observations on oxidative enzyme activity of glial cells in a local brain injury. J. Neuropath. exp. Neurol., 21, 116-136.

Strong, O. S., and Elwyn, A. (1953). Human Anatomy, 3rd edn. p. 45. Williams and Wilkins: Baltimore. 